University of Windsor

Scholarship at UWindsor

2008

\title{
A season-long team-building intervention: Examining the effect of team goal setting on cohesion
}

Julie Senecal

Todd M. Loughead

University of Windsor

Gordon A. Bloom

Follow this and additional works at: https://scholar.uwindsor.ca/humankineticspub

Part of the Kinesiology Commons, and the Sports Sciences Commons

\section{Recommended Citation}

Senecal, Julie; Loughead, Todd M.; and Bloom, Gordon A.. (2008). A season-long team-building intervention: Examining the effect of team goal setting on cohesion. Journal of Sport \& Exercise Psychology, 30 (2), 186-199.

https://scholar.uwindsor.ca/humankineticspub/18

This Article is brought to you for free and open access by the Faculty of Human Kinetics at Scholarship at UWindsor. It has been accepted for inclusion in Human Kinetics Publications by an authorized administrator of Scholarship at UWindsor. For more information, please contact scholarship@uwindsor.ca. 


\title{
A Season-Long Team-Building Intervention: Examining the Effect of Team Goal Setting on Cohesion
}

\author{
Julie Senécal, ${ }^{1}$ Todd M. Loughead, ${ }^{2}$ and Gordon A. Bloom ${ }^{1}$ \\ ${ }^{1}$ McGill University and ${ }^{2}$ University of Windsor
}

\begin{abstract}
The purpose of the current study was to determine whether the implementation of a season-long team-building intervention program using team goal setting increased perceptions of cohesion. The participants were 86 female high school basketball players from 8 teams. The teams were randomly assigned to either an experimental team goal-setting or control condition. Each participant completed the Group Environment Questionnaire (GEQ; Carron, Brawley, \& Widmeyer, 2002; Carron, Widmeyer, \& Brawley, 1985), which assessed cohesion at both the beginning and end of the season. Overall, the results revealed a significant multivariate effect, Pillai's trace $F(12,438)=2.68, p=.002$. Post hoc analyses showed that at the beginning of the season, athletes from both conditions did not differ in their perceptions of cohesion. However, at the end of the season, athletes in the team goal-setting condition held higher perceptions of cohesion than athletes in the control condition. Overall, the results indicated that team goal setting was an effective team-building tool for influencing cohesiveness in sport teams.
\end{abstract}

Keywords: team building, team goal setting, team cohesion

Cohesion has been defined as "a dynamic process that is reflected in the tendency for a group to stick together and remain united in the pursuit of its instrumental objectives and/or for the satisfaction of member affective needs" (Carron, Brawley, \& Widmeyer, 1998, p. 213). Not surprisingly, coaches are interested in enhancing the cohesiveness of their teams because it is believed that greater cohesion is related to improved performance and team success (Bloom, Stevens, \& Wickwire, 2003; Carron, Bray, \& Eys, 2002; Loughead \& Hardy, 2006). Carron, Colman, Wheeler, and Stevens (2002) determined the strength of the cohesion-performance relationship in their meta-analysis, which contained a total of 9,988 athletes from 1,044 teams. Overall, the results showed a moderate-to-large significant circular relationship between cohesion and performance in sport. Given this finding, it is not surprising that attempts have been made to enhance cohesion.

Senécal and Bloom are with the Department of Kinesiology and Physical Education, McGill University, Montreal, Quebec, Canada and Loughead is with the Department of Kinesiology, University of Windsor, Windsor, Ontario, Canada. 
The process of developing or enhancing cohesion can be accomplished through a process known as team building, which can be viewed as a method to "promote an increased sense of unity and cohesiveness and enable the team to function together more smoothly and effectively" (Newman, 1984, p. 27). That is, teambuilding interventions are designed to increase group effectiveness by enhancing group cohesiveness (Carron, Spink, \& Prapavessis, 1997). Given the importance of enhancing cohesion through team building, Carron and Spink (1993) advanced a conceptual model of the factors believed to enhance group cohesiveness. The conceptual model is linear in nature and comprises inputs, throughputs, and outputs. The inputs consist of team structure (e.g., role clarity, leadership, team norms) and team environment (e.g., proximity, distinctiveness). These two categories of inputs are assumed to influence the throughput of group processes (e.g., team goals, team sacrifices), which in turn influences the output, operationalized as group cohesion. Using this team-building model as a basis, sport psychology consultants (SPC) can deliver team-building interventions by focusing on factors believed to influence group cohesion. Although it is assumed that team-building interventions enhance cohesion, results from empirical studies have been equivocal. Some studies have found a positive increase in team cohesion (e.g., Carron \& Spink, 1993; Stevens \& Bloom, 2003; Voight \& Callaghan, 2001), whereas other studies have found no change in perceptions of cohesion (e.g., Bloom \& Stevens, 2002; Prapavessis, Carron, \& Spink, 1996).

Brawley and Paskevich (1997) identified several shortcomings with teambuilding research that might help to explain these equivocal findings. One of the shortcomings has been the use of the coach as the facilitator of the team-building interventions. As Brawley and Paskevich noted, coaches acting as intervention facilitator may not have the patience, time, commitment, and know-how to successfully facilitate a team-building intervention. As such, the current study used the SPC as the facilitator of the intervention. The rationale for this choice was also based on business and industry settings, in which the intervention specialist typically works directly with the work team (Buller, 1988; Buller \& Bell, 1986). In such settings, the intervention specialist emphasizes the empowerment of the work team members, and they are provided with greater insight into their company. Overall, it is believed that these employees would be more intrinsically motivated because of their increased competence and self-determination (Prapavessis et al., 1996).

A second shortcoming stated by Brawley and Paskevich (1997) was related to the research design. The authors noted that most team-building studies did not use a control group. Without the inclusion of a control group it is difficult to determine the impact of the team-building intervention. For example, Bloom and Stevens (2002) found no significant increase in cohesion as a result of their team-building intervention with an equestrian team. This begs the question as to whether the team-building intervention was beneficial. Consequently, the use of a control group would have reduced the number of rival hypotheses. Another methodological issue highlighted by Brawley and Paskevich concerned the duration of the team-building intervention. For instance, Prapavessis et al. (1996) found no changes between intervention (team building), placebo, and control groups in cohesion levels after administering their team-building intervention program over 8 weeks of a soccer season. It could be argued that the length of the intervention did not allow the long-term benefits of the team-building program to fully develop (Buller, 1988). 
More precisely, Brawley and Paskevich noted that team-building interventions in sport would generally require a minimum of a season to determine whether there were any meaningful changes. Therefore, the current study attempted to overcome these methodological problems by implementing the intervention over an entire season and by using a control group to determine whether changes in cohesion were attributable to the intervention.

A final issue influencing the results of previous team-building interventions has been the delivery of multiple intervention topics (Brawley \& Paskevich, 1997). Researchers have commonly introduced various types of interventions - role behavior, social support, team leadership, social interaction, and team goals - with the hope that one or more of these intervention topics would increase team cohesion (e.g., Bloom \& Stevens, 2002; Carron \& Spink, 1993; Prapavessis et al., 1996; Spink \& Carron, 1993; Stevens \& Bloom, 2003). However, if a team-building program is based on multiple interventions and the program enhances (or fails to) enhance cohesion, it becomes nearly impossible to determine which component(s) of the intervention program might have been successful (or unsuccessful).

The present study focused solely on the team-building intervention topic of team goal setting for several reasons. First, Stevens and Bloom (2003) found that athletes perceived this intervention topic to be the most effective in their teambuilding program. Second, Widmeyer and Ducharme (1997) noted that team goal setting can directly influence cohesion by encouraging a greater team focus. Third, goal-setting research has grown exponentially in the last two decades (Burton, Nalylor, \& Holliday, 2001). Although the majority of research has been on individual goal setting, research on team goal setting has also provided evidence that it contributes to team outcomes. For example, Brawley, Carron, and Widmeyer (1993) found that team goals positively influenced team satisfaction, cohesion, and performance. Furthermore, Locke and Latham (1990) indicated that 38 out of 41 group goal-setting studies found a positive performance enhancement effect. Interestingly, the authors noted group goals had virtually the same success rate as individual goal setting findings.

Therefore, the purpose of the current study was to determine whether the implementation of a season-long team-building program using team goal setting would increase perceptions of cohesion compared with a control condition receiving no treatment over the course of an entire regular season. The current intervention program was implemented and facilitated by a SPC and used only one intervention tool-team goal setting. Before the start of the team goal-setting intervention, it was hypothesized that athletes in both conditions (team goal setting and control) would be similar on perceptions of cohesion; however, after participating in a season-long team goal-setting program, athletes in this condition would have higher perceptions of cohesion than their control counterparts.

\section{Method}

\section{Participants}

A total of 86 female high school senior basketball players from eight teams served as participants. The eight teams competed at the girls Juvenile level (players ranging in age from 14 to 18 years) in one of three divisions, based on school size. 
The mean age of the athletes was 15.71 years $(S D=.96)$. The athletes played on average 1.3 years on their current team and had played organized basketball for approximately 5 years. Each team practiced at least two times per week and played one game per week over the course of the 5-month regular season. Four teams were randomly placed into the team goal-setting experimental condition and four into the no-treatment control condition. Teams in the team goal-setting condition were involved in the intervention program over the course of the regular season. On the other hand, teams in the control condition were run as a traditional sport team with no sport psychology interventions.

\section{Experimental Conditions}

Team Goal-Setting Condition. Eys, Patterson, Loughead, and Carron's (2006) three-stage protocol for implementing a team goal-setting program was used in the current study (see Table 1 for a brief overview). In the first stage, the rationale for the intervention and the setting of team goals was discussed and carried out with the athletes. As Gould (2001) suggested, coaches and athletes need more education about goal setting and the benefits of this type of intervention. They were informed that working together to find common objectives for their team could help them work better as a unit. As a team, the athletes generated appropriate long- and shortterm outcome goals. Typically, the former is reflected in overall team standing (e.g., finish among the top two teams in the league), and the latter is reflected in outcomes

\section{Table 1 A Brief Overview of the Team Goal-Setting Program}

\begin{tabular}{|c|c|}
\hline Principle & Implementation strategy \\
\hline Selecting the team goals. & $\begin{array}{l}\text { 1. Athletes are provided with a list of performance indices (e.g., } \\
\text { turnovers, steals, blocked shots, free throw shooting percentage). } \\
\text { 2. From the list, each athlete independently identifies the four most } \\
\text { important performance indices to establish for team goals. } \\
\text { 3. Subunits of five athletes agree on the four most important } \\
\text { performance indices that should be set for team goals. } \\
\text { 4. The team as whole then agrees on the four performance indices } \\
\text { that team goals will be set. }\end{array}$ \\
\hline
\end{tabular}

Establishing the target for the team goals.

Coaches remind players of the team's goals.

Evaluation, feedback, and reevaluation are essential for team goalsetting effectiveness.
1. Each athlete independently identifies the target levels for each of the four team outcomes.

2. Subunits of five athletes agree on the target levels.

3 . The team as a whole then agrees on the target levels.

The goals are posted in the team's locker room.

The sport psychology consultant meets with the team to review and discuss the team's goals after each block of three games. Modifications to the team's goals or target levels were made if deemed necessary. 
in an upcoming series of games (e.g., obtain two wins in the next three games). Once the long- and short-term goals were established, the following question was asked by the SPC: "What do you have to do especially well as a team on a gameto-game basis to maximize your chances of reaching your short- and long-term goals?" Athletes were then provided with a list of approximately 10 performance (game) indices that were specific and measurable (e.g., rebounds, turnovers). Each athlete independently picked four performance indices (i.e., performance goals) that she thought were most important for her team. Athletes were then assigned to subgroups of five individuals and asked to discuss and negotiate until consensus on four performance goals was obtained. Initially working in smaller groups prior to working with the total team increased the likelihood that each player's views were considered. Finally, the performance goals emanating from each of the subgroups were discussed with the whole team. The four performance goals considered most important were then established by the entire team.

Once these four team goals were decided, the target to strive for in each game was established. To this end, athletes were provided with statistics from the previous season. Following this, the process previously described was repeated. First, each athlete independently determined the target she believed to be appropriate (e.g., obtain $55 \%$ of free throws as a team). Then, the athletes were assigned to the same subgroup of five people to discuss and negotiate appropriate target levels for each of the team goals previously chosen. Finally, the target levels chosen in each subgroup were discussed as a team and a team decision was made for each team goal.

In the second stage, coaches were instructed to remind their players of the team's goals along with their target levels before each game. Results for each team goal were posted after every game by the coach for the athletes to examine. In the third stage, the SPC met with the team to review and discuss the goals after each block of three games. At this meeting, modifications to the team goals were made by adding goals, removing goals, and/or changing the target levels if necessary. If alterations to the team goals were required, the procedure described in the first stage was repeated.

Control Condition. The teams in the control condition were informed that they would complete an inventory measuring cohesion twice during the season-once at the beginning of the season and once at the end of the season. Throughout the season, the teams in this condition received no additional information from the SPC.

\section{Measures}

Cohesion. Cohesion was measured using the Group Environment Questionnaire (GEQ; Carron, Widmeyer, \& Brawley, 1985; Carron, Brawley, \& Widmeyer, 2002). The GEQ is an 18-item inventory that assesses four dimensions of cohesion: Group Integration-Task (GI-T), Group Integration-Social (GI-S), Individual Attractions to the Group-Task (ATG-T), and Individual Attractions to the Group-Social (ATG-S). An example item for the GI-T dimension was, "Our team is united in trying to reach its goals for performance." As for the GI-S dimension, an example item was, "Our team would like to spend time together in the off season." An example item for the ATG-T dimension was, "I do not like the style of play on this team." For the ATG-S dimension, an example item was, "Some of my best friends are on this team." Responses were anchored on a 9-point Likert scale by 1 (I strongly 
disagree) to 9 (I strongly agree). Thus, higher scores reflected higher perceptions of cohesion. Research using the GEQ has provided evidence that the scores obtained from the four dimensions are both valid and reliable. More specifically, research has shown that the scores from the four dimensions as represented by the GEQ are internally consistent and demonstrate content, predictive, and concurrent validity (Carron et al., 1998).

Postintervention Manipulation Check. At the end of the season, two manipulation checks were performed: one for the team goal-setting condition and one for the control condition. On the one hand, athletes in the team goal-setting condition were asked to anonymously complete a five-item open-ended questionnaire to help evaluate the effectiveness of the program. The nature of the questions was based on Bloom and Stevens's (2002) sport psychology evaluation form. Questions included, "Did the team goal setting program help your team play better together?" and the follow-up question, "How did the team goal setting program help your team play better?" On the other hand, athletes in the control condition answered a brief questionnaire to assess whether team-building activities were implemented by coaches. For instance, questions included, "Throughout your regular season, were there any activities implemented by your coach to affect the closeness of your team either on or off the court?" and "How do you perceive your team's closeness at the end of the season?" This manipulation check helped understand whether and to what extent the coaches in the control condition may have influenced team cohesion.

Team Tenure. Team tenure was operationalized as the number of years the athletes had been a member of their current team as self-reported by the participants.

Playing Experience. This construct was operationalized as the number of years the participants had been playing organized basketball.

Team Performance. Performance was assessed using a team's winning percentage at the end of the regular season.

\section{Procedures}

All teams ( $n=17$ teams) from a girls high school basketball league were initially invited to take part in a study examining cohesion in female basketball players. From this pool of 17 teams, eight coaches voluntarily agreed to participate in this study. Teams were then randomly assigned to either a team goal-setting condition or a control condition. In particular, teams in the team goal-setting condition would be involved in a season-long intervention program and would complete the GEQ on two occasions (Time 1, beginning of the season; Time 2, end of the regular season) in addition to a team goal-setting evaluation inventory at season's end. Teams in the control condition would complete the GEQ twice (same as the team goal-setting condition) and a brief questionnaire at the end of the season. Data collection took place before or after a training session at the teams' practice facility. The SPC implemented and monitored teams in the team goal-setting condition. The team goal-setting sessions occurred prior to the start of a practice at the team's facility. These sessions took approximately 20-30 min to complete. In addition, information was also collected on team tenure, playing experience, and team performance. Finally, ethical approval for the study was obtained from 
the university's research ethics board and the school board's athletic association. In addition, informed consent was obtained from the players' parents and assent from the players.

\section{Data Analysis}

Academics have recently used two approaches when dealing with individual-level responses in group-level constructs. On the one hand, some authors (e.g., Bliese, Halverson, \& Schriesheim, 2002; Lance, Butts, \& Michels, 2006) advocate that "it is generally accepted that demonstration of sufficient within-group agreement is a necessary precondition for the aggregation of more micro-level (e.g., individuallevel) measures to represent more macro-level (e.g., group-level) constructs (Lance et al., p. 207). On the other hand, some researchers (e.g., Myers \& Feltz, 2007; Watson, Chemers, \& Preiser, 2001) feel that multilevel software programs (e.g., HLM) do not require an acceptable level of consensus to calculate an average of the individual responses within each team. Furthermore, Myers and Feltz noted that what constitutes an acceptable level of consensus has not been established.

Table 2 shows that team affiliation was a significant predictor of the four dimensions of cohesion as indicated by the significant $F$ ratios. The intraclass correlation (ICC) ranged between .12 and .44 , and the consensus (index of agreement, $r_{\text {wg(j) }}$ ) values ranged from .49 to .55 . Based on these values, authors such as Bliese et al. (2002) and Lance et al. (2006) would suggest that the ICC values are somewhat low, indicating an individual-level variability in the scores. As well, the low $r_{\mathrm{wg}(\mathrm{j})}$ values would suggest minimal agreement. Taken together, these teams should not have their individual level scores aggregated. Despite this, authors such as Myers and Feltz (2007) would recommend a multilevel approach because nested effects in designs (i.e., athletes within teams) create some undesirable consequences: failure to account for important sources of variances and violation of independence of observations. As a result, using traditional analyses such as MANOVA could lead to a higher probability of inappropriately rejecting the null hypothesis. Given that there were only eight teams in the current study, we were constrained by low statistical power, and as Pollack (1998) noted an ANOVA technique may be more appropriate when sample sizes are modest. For this reason, individual-level analyses were conducted. Thus, the analyses that follow should be interpreted with this caveat in mind.

\section{Table 2 The Estimates of Nonindependence, Reliability, and} Agreement for Each Dimension of Cohesion

\begin{tabular}{lccc}
\hline Dimension & $\boldsymbol{F}$ ratio & ICC & $\boldsymbol{r}_{\text {wg(j) }}$ \\
\hline ATG-T & $4.84 *$ & .26 & .51 \\
ATG-S & $3.55^{*}$ & .19 & .49 \\
GI-T & $2.43^{*}$ & .12 & .50 \\
GI-S & $9.34 *$ & .44 & .55 \\
\hline
\end{tabular}

Note. ATG-T = individual attractions to the group - task; ATG-S = individual attractions to the group - social; GI-T = group integration - task; GI-S = group integration - social.

$* p<.05$. 


\section{Results}

\section{Descriptive Statistics}

Internal consistency estimates were computed for each of the four GEQ dimensions at both Time 1 (at the beginning of the regular season) and Time 2 (near the end of the regular season). Cronbach's alpha values for all the dimensions were acceptable based on Nunally's (1978) recommendations (ATG-T, Time 1, $\alpha=.80$, Time 2, $\alpha=.86$; ATG-S, Time 1, $\alpha=.76$, Time 2, $\alpha=.87$; GI-T, Time $1, \alpha=.72$, Time 2, $\alpha=.85$; and GI-S, Time $1, \alpha=.70$, Time $2, \alpha=.71$ ).

A summary of the descriptive statistics can be found in Table 3. Of note, participants in the team goal-setting condition had higher perceptions of cohesion after completing the season-long intervention (Time $1 \mathrm{vs}$. Time 2 ). In contrast, the control group had a decrease in perceptions of cohesion from the beginning to the end of the regular season on all dimensions (Time 1 vs. Time 2). Finally, it was shown that at the end of the season the team goal-setting condition had greater perceptions of cohesion than the control group for all dimensions of cohesion. A summary of the bivariate correlations among the variables can be found in Table 4 . Significant Pearson correlation coefficients for the dimensions of cohesion ranged from $r=.321$ to $r=.659$ at Time 1. As for Time 2, significant Pearson correlation coefficients ranged from $r=.548$ to $r=.809$, providing some evidence that multicollinearity was minimal (Tabachnick \& Fidell, 2001).

\section{Preliminary Analyses}

Before undertaking the main analyses, a MANOVA was conducted to determine whether team tenure, playing experience, and team performance differed between the experimental conditions. On the one hand, the results indicated that the team goal-setting condition had a team tenure of 1.18 years, basketball playing experience of 5.38 years, and a winning percentage of 67.5. On the other hand, the control condition had a team tenure of 1.40 years, basketball playing experience of 4.90 years, and a winning percentage of 55.5.

Table 3 Means and Standard Deviations of Cohesion for the Team GoalSetting Condition and Control Condition at Times 1 and 2

\begin{tabular}{|c|c|c|c|c|}
\hline \multirow[b]{2}{*}{ Dimension } & \multicolumn{2}{|c|}{ Time 1} & \multicolumn{2}{|c|}{ Time 2} \\
\hline & $\begin{array}{c}\text { Team Goal Setting } \\
M(S D)\end{array}$ & $\begin{array}{l}\text { Control } \\
M(S D)\end{array}$ & $\begin{array}{c}\text { Team Goal Setting } \\
M(S D)\end{array}$ & $\begin{array}{l}\text { Control } \\
M(S D)\end{array}$ \\
\hline ATG-T & $6.77(1.23)$ & $7.03(1.67)$ & $6.72(1.80)$ & $5.88(2.18)$ \\
\hline ATG-S ${ }^{a}$ & $6.50(1.82)$ & $6.23(1.41)$ & $6.71(1.63)$ & $5.31(1.91)$ \\
\hline GI-T $^{a}$ & $6.06(1.34)$ & $5.76(1.44)$ & $6.63(1.69)$ & $5.32(1.60)$ \\
\hline GI-S & $6.22(1.30)$ & $6.05(1.82)$ & $6.47(1.44)$ & $5.06(2.15)$ \\
\hline
\end{tabular}

Note. ATG-T = individual attractions to the group - task; ATG-S = individual attractions to the group - social; GI-T = group integration - task; GI-S = group integration - social.

${ }^{\mathrm{a}}$ Assessed on a 9-point scale ranging from 1 to 9 . 
Table 4 Bivariate Correlations Between the Dimensions of Cohesion at Times 1 and 2

\begin{tabular}{lcccc}
\hline & $\mathbf{1}$ & $\mathbf{2}$ & $\mathbf{3}$ & $\mathbf{4}$ \\
\hline & & Time 1 & & \\
1. ATG-T & - & $.321^{* *}$ & $.421^{* *}$ & $.640^{* *}$ \\
2. ATG-S & & - & $.606^{* *}$ & $.495^{* *}$ \\
3. GI-T & & & - & $.659^{* *}$ \\
4. GI-S & & Time 2 & & - \\
1. ATG-T & $-548^{* *}$ & $.772^{* *}$ & $.786^{* *}$ \\
2. ATG-S & & - & $.663^{* *}$ & $.704^{* *}$ \\
3. GI-T & & & - & $.809^{* *}$ \\
4. GI-S & & & & - \\
\hline
\end{tabular}

Note . ATG-T $=$ individual attractions to the group - task $;$ ATG-S $=$ individual attractions to the group social; GI-T = group integration - task; GI-S = group integration - social.

**Correlation significant at the .01 level.

The results of the MANOVA revealed a nonsignificant main effect for experimental conditions, Pillai's trace, $F(1,130)=.86, n s$. That is, the results indicated that team tenure, playing experience, and performance were no different between the team goal-setting and control conditions.

\section{Main Analyses}

A MANOVA with univariate follow-up tests was conducted to address the study's primary purpose, which was to determine whether athletes in a season-long teambuilding intervention using team goal setting experienced greater perceptions of cohesion than a control group at the end of the season. The MANOVA revealed a significant multivariate effect, Pillai's trace $F(12,438)=2.68, p<.05$, and univariate analyses demonstrated that the groups differed significantly in perceptions of cohesion on all four dimensions: ATG-T, $F(3,147)=2.90, p<.05$; ATG-S, $F(3$, $147)=4.61, p<.05$; GI-T, $F(3,147)=4.82, p<.05$, and GI-S, $F(3,147)=4.48$, $p<.05$. Three planned comparison post hoc analyses using the Tukey-Kramer procedure were conducted. The first post hoc analyses examined whether there were any differences in cohesion levels between the team goal-setting condition and the control condition at the beginning of the season (i.e., Time 1). The results showed no significant difference in cohesion (ATG-T, ATG-S, GI-T, GI-S) between the individuals in the team goal-setting condition and the control group at Time $1(p>.05)$.

The second post hoc analysis examined whether there were any differences in cohesion levels between the team goal-setting condition and the control condition 
at the end of the season (i.e., Time 2). The results of the post hoc showed that at the end of the season, athletes in the team goal-setting condition had higher perceptions of cohesion on all four dimensions than athletes in the control condition $(p<$ $.05)$. In an attempt to explain the changes in cohesion levels that occurred over the course of the season, the third post hoc examined whether these changes in cohesion occurred in the team goal-setting condition or in the control condition. The results revealed that individuals in the team goal-setting condition did not significantly increase their level of cohesion on any of the four dimensions (ATG-T, ATG-S, GI-T, GI-S) between the two time periods - beginning of the season vs. end of the season $(p>.05)$. However, athletes in the control condition showed a significant decrease in cohesion from the start of the season to the end of the season on three (i.e., ATG-T, ATG-S, GI-S) of the four dimensions $(p<.05)$.

\section{Postintervention Manipulation Check}

Team Goal-Setting Condition. The majority of players $(68.4 \%)$ responded that the team-building intervention helped them play better together as a team. When examining why, the players mentioned that it enabled them to be more focused on common goals $(42.3 \%)$, allowed them to work together to reach their goals $(26.9 \%)$, forced them to work harder $(11.5 \%)$, and helped them set more realistic and manageable goals $(7.7 \%)$. A small portion of the players $(3.8 \%)$ indicated the program helped them to be more organized and to communicate better, it motivated them to reach their goals, and it brought the team's shortcomings to the forefront.

Control Condition. The manipulation check for the control condition identified whether and to what extent the coaches may have influenced the development of team cohesion. The results indicated the coaches in the control condition implemented a variety of one-time activities. For example, coaches occasionally had team outings to the movies or team dinners. They also encouraged players to cheer for one another. Some of the coaches had a few team meetings to develop trust between the athletes. Taken together, it was concluded that no systematic team-building activities or team goal-setting intervention was implemented by the coaches in the control condition.

\section{Discussion}

The purpose of this study was to determine whether the implementation of a season-long team-building intervention using team goal setting increased perceptions of cohesion. Specifically, it was hypothesized that athletes in both conditions (team goal setting and control) would be similar on perceptions of cohesion before the intervention. However, after participating in a season-long team goal-setting program, athletes in this condition would have higher perceptions of cohesion than their control counterparts. In general, the results supported our hypotheses. First, the results showed that athletes from both conditions were similar on all four dimensions of cohesion before the team goal-setting intervention. Second, after completing the season-long team goal-setting intervention, athletes from this condition perceived levels of cohesion higher than those of the control athletes on 
all four dimensions. Beyond these findings, a number of aspects associated with the results should be highlighted.

The findings of the current study highlighted the importance of team goal setting not so much for enhancing cohesion but as a method of, at least, maintaining it for the duration of the season. More specifically, the results indicated that athletes in the team goal-setting condition did not significantly increase perceptions of cohesion over the course of the season. That is, the level of cohesion for athletes in the team goal-setting condition remained stable. In contrast, at the end of the regular season athletes in the control condition had a significant decrease in perceptions of cohesion compared with at the start of the season on three of the four dimensions (ATG-T, ATG-S, GI-S). These results may be attributed to a ceiling effect. Given that the athletes already perceived a high level of cohesion at the start of the season, perhaps it is not surprising that cohesion levels did not increase at the end of the season for those in the team goal-setting condition. However, because the control condition showed a decrease in cohesion, the results of the current study are in line with the suggestion that athletes participating in team-building interventions demonstrate greater task and social cohesion (Brawley et al., 1993; Stevens \& Bloom, 2003).

The results of the current study suggested that both individual and group orientations of cohesion are important when implementing a team-building program. Stevens and Bloom (2003) purposely measured the group-oriented dimensions of cohesion (i.e., GI-T and GI-S), which they attributed to the group-oriented nature of their team-building activities. However, the current results showed that athletes completing the team goal-setting program perceived higher levels of cohesion on all four dimensions in comparison with athletes in the control condition. This indicates that all dimensions of cohesion are important when conducting team-building activities. That is, even individual dimensions of cohesion (i.e., ATG-T, ATG-S) are important when conducting group activities such as team goal-setting programs. This could be a function of the protocol used in the current study whereby each athlete first had to individually identify important team goals, before discussing them with the group as a whole. Therefore, by having the athletes develop the team goals individually, the protocol may have helped enhance each team member's feelings about her own personal involvement with the group's task and goal (i.e., ATG-T) and each team member's feelings about her personal acceptance, and social interaction with the group (i.e., ATG-S).

It is worth noting that the current team goal-setting program exclusively emphasized task aspects, such as reducing the number of turnovers and increasing the number of rebounds during basketball games. However, the results of the current study found that social cohesion was greater for the athletes in the team goal-setting condition compared with their control counterparts. This finding supports Tziner, Nicola, and Rizac's (2003) contention that a positive perception of social cohesion may evolve when collaborative interactions between team members are encouraged through the development of task strategies (e.g., team goals).

The current study attempted to overcome two methodological limitations of previous team-building intervention studies (cf. Brawley \& Paskevich, 1997). More specifically, we used a control group and we used only one team-building intervention topic (i.e., team goal setting). By using a control group, we were able to determine that the team-building intervention was effective. In particular, the results 
indicated that at the beginning of the season athletes from both conditions did not differ in their perceptions of cohesion. However, athletes in the team goal-setting condition had higher perceptions of cohesion at the end of the season compared with their control condition counterparts. Perhaps the use of a control group would have allowed Bloom and Stevens (2002) to quantitatively demonstrate the value of their team-building intervention program in the same manner as the current study. As for the use of only one intervention, team goal setting, the results of the current study highlight the usefulness of this intervention. In particular, having team members participate in a team goal-setting program may facilitate cohesion because it involves cooperation among teammates. As a result, team members learn that to achieve the team's goals they must depend on each other (Widmeyer \& Ducharme, 1997). As Brawley et al. (1993) noted, when teams perform a task together, such as team goal setting, perceptions of cohesion are enhanced.

In an effort to understand the contributions of team-building interventions, the current study implemented postintervention manipulation checks for both conditions. On the one hand, the results of the manipulation checks showed that athletes in the control condition felt their coaches did not implement any systematic teambuilding interventions. Only one-time activities such as team dinners or outings to the movies were reported. This result lends support to Prapavessis et al.'s (1996) contention that teams will naturally undertake a variety of social activities that the coach believes will increase team unity. However, knowing that cohesion levels decreased throughout the season for the control condition despite some minor efforts by the coaches to enhance group unity, it would appear that these types of minor efforts are not sufficient to enhance or maintain cohesion levels throughout the season. On the other hand, the postmanipulation check of the team goal-setting condition appears to highlight the effectiveness of the intervention. Specifically, the results indicated that the team-building program was perceived as being effective for helping the athletes play better by developing common team goals. This result is consistent with the notion advanced by Cox (1990) that team goal setting can motivate players to work toward a common goal. The results of the current study also appear to confirm the suggestion of Widmeyer and Ducharme (1997) that team goal setting can influence cohesion by encouraging a greater team focus.

Although the results of the current study are promising, future research is still required. First, future studies should examine other types of sports. As Carron et al. (2002) found, cohesion has been associated with performance in both interactive team sports (e.g., basketball) and coactive team sports (e.g., swimming). Furthermore, Carron et al. stated that players from coactive team sports have fewer opportunities for team cohesion to develop; therefore, having these types of teams involved in team-building activities, such as team goal setting, may have a substantial impact on cohesion. Future research could compare whether there are differences between these two types of sports. Whereas the current study focused solely on task aspects in regard to team goal setting, the results showed that the intervention impacted social cohesion. Consequently, future research could examine whether a team-building intervention focused on only social aspects would impact both task and social cohesion. As well, the current study measured cohesion at two time points in the season. As a result, we were unable to track the trajectory of cohesion over time. It is recommended that future studies measure cohesion at multiple time periods throughout the season to determine at which point changes 
in cohesion occur in both experimental and control groups. Finally, Carron et al. found that gender influenced the cohesion-performance relationship. In particular, research has shown that perceptions of cohesion in female athletes are more related to performance than in male athletes. Thus, future research should also examine the effect of team goal setting on male athletes.

To summarize, this study attempted to build upon a small body of research on team building. In particular, the study was designed so that a number of previous methodological shortcomings were addressed. The results indicated support for the use of a team-building intervention focusing on team goal setting. The results suggest that the Eys et al. (2006) protocol is a viable option for those interested in implementing a systematic team goal-setting program. Addressing some of the methodological concerns and the use of a systematic team goal-setting protocol will hopefully encourage researchers to continue developing, refining, and designing team-building interventions.

\section{References}

Bliese, P.D., Halverson, R.R., \& Schriesheim, C.A. (2002). Benchmarking multilevel methods of leadership: The articles, the model, and the data set. Leadership Quarterly, 13, 3-14.

Bloom, G.A., \& Stevens, D.E. (2002). A team building mental skills training program with an intercollegiate equestrian team. Athletic Insight, 4, www.athleticinsight.com/Vol4Iss1/ EquestrianTeamBuilding.htm.

Bloom, G.A., Stevens, D.E., \& Wickwire, T.L. (2003). Expert coaches' perceptions of team building. Journal of Applied Sport Psychology, 15, 129-143.

Brawley, L.R., Carron, A.V., \& Widmeyer, W.N. (1993). The influence of the group and its cohesiveness on perceptions of group goal-related variables. Journal of Sport \& Exercise Psychology, 15, 245-260.

Brawley, L.R., \& Paskevich, D.M. (1997). Conducting team building research in the context of sport and exercise. Journal of Applied Sport Psychology, 9, 11-40.

Buller,P.F. (1988). Long term performance effects of goal setting and team building interventions in an underground silver mine. Organization Development Journal, 6, 82-93.

Buller, P.F., \& Bell, C.H. (1986). Effects of team building and goal setting on productivity: A field experiment. Academy of Management Journal, 29, 305-328.

Burton, D., Nalylor, S., \& Holliday, B. (2001). Goal setting in sport: Investigating the goal effectiveness paradox. In R.N. Singer, H.A. Hausenblas, \& C.M. Janelle (Eds.), Handbook of sport psychology (2nd ed., pp. 497-528). New York: Wiley.

Carron, A.V., Brawley, L.R., \& Widmeyer, W.N. (1998). Measurement of cohesion in sport and exercise. In J.L. Duda (Ed.), Advances in sport and exercise psychology measurement (pp. 213-226). Morgantown, WV: Fitness Information Technology.

Carron, A.V., Brawley, L.R., \& Widmeyer, W.N. (2002). The Group Environment Questionnaire test manual. Morgantown, WV: Fitness Information Technology.

Carron, A.V., Bray, S.R., \& Eys, M.A. (2002). Team cohesion and team success in sport. Journal of Sports Sciences, 20, 119-126.

Carron, A.V., Colman, M.M., Wheeler, J., \& Stevens, D. (2002). Cohesion and performance in sport: A meta-analysis. Journal of Sport \& Exercise Psychology, 24, 168-188.

Carron, A.V., \& Spink, K.S. (1993). Team building in an exercise setting. The Sport Psychologist, 7, 8-18.

Carron, A.V., Spink, K.S., \& Prapavessis, H. (1997). Team building and cohesiveness in the sport and exercise setting: Use of indirect interventions. Journal of Applied Sport Psychology, 9, 61-72. 
Carron, A.V., Widmeyer, W.N., \& Brawley, L.R. (1985). The development of an instrument to assess cohesion in sport teams: The Group Environment Questionnaire. Journal of Sport Psychology, 7, 224-266.

Cox, R.H. (1990). Sport psychology: Concepts and application (2nd ed.). Madison, WI: Brown \& Benchmark.

Eys, M.A., Patterson, M.M., Loughead, T.M., \& Carron, A.V. (2006). Team building in sport. In J. Duda, D. Hackfort, \& R. Lidor (Eds.), Handbook of research in applied sport psychology: International perspectives (pp. 219-231). Morgantown, WV: Fitness Information Technology.

Gould, D. (2001). Goal setting for peak performance. In J.M. Williams (Ed.), Applied sport psychology: Personal growth to peak performance (4th ed., pp. 190-205). Mountain View, CA: Mayfield.

Lance, C.E., Butts, M.M., \& Michels, L.C. (2006). The sources of four commonly reported cutoff criteria: What did they really say? Organizational Research Methods, 9, 202-220.

Locke, E.A., \& Latham, G.P.(1990). A theory of goal setting and task motivation. Englewood Cliffs, NJ: Prentice-Hall.

Loughead, T.M., \& Hardy, J. (2006). Team cohesion: From theory to research to team building. In S. Hanton \& S. Mellalieu (Eds.), Literature reviews in sport psychology (pp. 257-287). Hauppauge, NY: Nova Science Publishers.

Myers, N.D., \& Feltz, D.L. (2007). From self-efficacy to collective efficacy in sport: Transitional methodological issues. In G. Tenenbaum \& R.C. Eklund (Eds.), Handbook of research on sport psychology (3rd ed., pp. 799-819). Hoboken, NJ: Wiley.

Newman, B. (1984). Expediency as benefactor: How team building saves time and gets the job done. Training and Development Journal, 38, 26-30.

Nunally, J.C. (1978). Psychometric theory (2nd ed.). New York: McGraw-Hill.

Pollack, B.N. (1998). Hierarchical linear modeling and the "unit of analysis" problem: A solution for analyzing responses of intact group members. Group Dynamics. Theory, Research, and Practice, 2, 299-312.

Prapavessis, H., Carron, A.V., \& Spink, K.S. (1996). Team building in sport. International Journal of Sport Psychology, 27, 269-285.

Spink, K.S., \& Carron, A.V. (1993). The effects of team building on the adherence patterns of female exercise participants. Journal of Sport \& Exercise Psychology, 15, 39-49.

Stevens, D.E., \& Bloom, G.A. (2003). The effect of team building on cohesion. Avante, 9, 43-54.

Tabachnick, B.G., \& Fidell, L.S. (2001). Using multivariate statistics (4th ed.). Boston: Allyn and Bacon.

Tziner, A., Nicola, N., \& Rizac, A. (2003). Relation between social cohesion and team performance in soccer teams. Perceptual and Motor Skills, 96, 145-148.

Voight, M., \& Callaghan, J. (2001). A team building intervention program: Application and evaluation with two university soccer teams. Journal of Sport Behavior, 24, 420-431.

Watson, C.B., Chemers, M.M., \& Preiser, N. (2001). Collective efficacy: A multilevel analysis. Personality and Social Psychology Bulletin, 27, 1056-1068.

Widmeyer, W.N., \& Ducharme, K. (1997). Team building through team goal setting. Journal of Applied Sport Psychology, 9, 97-113.

Manuscript submitted: May 16, 2007

Revision accepted: December 1, 2007 